www.nature.com/clinicalpractice/uro

\title{
Use of antibiotics in pediatric urology
}

\author{
Alexandra M Hay
}

The emergence of antibiotic-resistant strains of bacteria and how to treat their associated infections are major challenges in medicine today. With frequent media reports on the rising incidence of "superbugs", such as methicillinresistant Staphylococcus aureus and multiple drug-resistant tuberculosis, limiting the prescription of antibiotics and ensuring that patients on antibiotics comply fully with treatment regimens are very important issues.

A study in the BMJ (Chung A et al. [2007] BMJ [doi:10.1136/bmj.39274.647465.BE]) examining the prescribing of antibiotics to children in primary care highlights these issues. The authors examined a population of children attending their primary care physician for respiratory infections. They found that children prescribed an antibiotic had more than double the risk of harbouring antibiotic-resistant bacteria in throat swabs taken at 2-week follow up compared with those not treated. Although the number of resistant bacteria fell back to normal levels at 12-week follow up, the authors suggest that current prescribing patterns could be enough to sustain a high level of antibiotic resistance in the population. This is of great concern for public health and it is clear that the use of antibiotics needs to be scrutinized more closely.

In urology, the pediatric patient population is prescribed antibiotics for a whole range of different medical problems. In this issue of Nature Clinical Practice Urology, Hensle and colleagues discuss the use of routine antibiotic prophylaxis in children with vesicoureteral reflux (VUR) (Hensle et al. [2007] Nat Clin Pract Urol 4: 462-463). They challenge the clinical practice guidelines that recommend children with VUR should be routinely prescribed prophylactic antibiotics in order to reduce the chances of developing urinary tract infection (UTI) and renal scarring. Whilst the guidance itself isn't necessarily at fault, the biggest problem in this patient population is compliance. In a study by the authors (currently in press) the overall "....current

prescribing

patterns could

be enough

to sustain

a high level

of antibiotic

resistance

in the

population."

AM Hay is Editor of Nature Clinical Practice Urology.

Competing interests

The author declared no competing interests

www.nature.com/clinicalpractice doi:10.1038/ncpuro0908 treatment compliance was poor, with only $10 \%$ of children having antibiotic coverage during a 12-month period. In the same study it was reported that $64 \%$ of patients classified as being $100 \%$ compliant with antibiotic treatment had at least one diagnosis of a UTI. This finding raises a question about whether continuous lowdose antibiotic prophylaxis really does achieve the aim of reducing UTIs and subsequent renal scarring in this population.

This issue of prescribing prophylactic antibiotics to children with VUR has also been raised by Conway and colleagues (Conway $\mathrm{PH}$ et al. [2007] JAMA 298:179-186) in their study of recurrent UTIs in children in a primary care setting. The authors studied the incidence of recurrent UTIs in children up to 6 years of age and recorded the use of antibiotics and levels of bacterial resistance. Associations were found between older age (3 to 5 years), white race and presence of grade 4-5 VUR and an increased risk of recurrent UTIs. Interestingly, antimicrobial prophylaxis did not reduce the risk of children developing recurrent infections but it was associated with an increased risk of antibiotic resistance. Race might also play a role, as in this study nonwhite children were more likely to have resistant infections, despite them being less likely to have recurrent UTIs, than white children.

Although these studies highlight important issues surrounding the use of antibiotics in children for both urologic and other indications, antibiotics still play an essential role in the treatment of many urologic diseases in the pediatric population. However, if we are to reduce the levels of antibiotic resistance it is clear that their use needs to be carefully scrutinized. Important factors to consider include appropriate selection of patients and which agent to use, along with the dose, route of administration and duration of treatment. Strategies aimed at improving compliance with treatment regimens should also be examined in order to reduce the emergence of antibiotic-resistant bacteria. 